\title{
Studying Trapped Grains in Alumina using SEM and EBSD
}

\author{
J. L. Riesterer, J. K. Farrer,* N. Ravishankar, $\uparrow$ and C. B. Carter \\ Dept. of Chemical Engineering and Materials Science, University of Minnesota, 151 Amundson \\ Hall, 421 Washington Ave. SE, Minneapolis, MN 55455 \\ *Dept. of Physics and Astronomy, N283 ESC, Brigham Young University, Provo, UT 84602 \\ $\uparrow$ Materials Science Centre, Indian Institute of Science, Bangalore, India
}

Despite development of high-resolution [1] and in situ [2] microscopies, grain boundaries remain something of an enigma, especially in ceramic materials where second-phase glass is often found to be present as films or precipitates in the interfacial regions. The glass is an unavoidable product in the liquid-phase sintering (LPS) process and incorporates intentional additives and accidental impurities. [3-4] Abnormal grain growth (AGG) has been associated with second-phase dissolution rather than defect or pore coalescence. [5-6] The number of crystal defects inside the polycrystalline material may dictate which of the grains will grow and which will be consumed. [5] Crystal anisotropy will promote orientation-dependent lattice diffusion of solutes, which may result in an induced strain energy within the crystal. [7] The solutes will lower the strain energy by sitting at the spacious boundaries rather than in the grain matrix.

The effect of misorientation on grain-boundary migration has been monitored in the present study using electron backscatter diffraction (EBSD). Single-crystal alumina has been hot-pressed to a pure polycrystalline alumina slab at $1650^{\circ} \mathrm{C}$ for $30 \mathrm{~min}, \mathrm{~A} \mathrm{CaAl}_{2} \mathrm{Si}_{2} \mathrm{O}_{8}$ glass film, deposited via pulsed laser deposition (PLD), was used to enhance LPS and promoted AGG in the polycrystalline region. As a result, AGG of the polycrystalline alumina section occurred rather than normal grain growth. One interesting feature of the observed AGG was the presence of smaller abnormal grains trapped within huge abnormal grains. Grain-growth theory [8] would predict that larger grains should consume smaller grains. Instead, some of the trapped grains appear to be remarkably stable inside the large grain matrix. Grain $X$ in figure 1a was analyzed using EBSD. Remnant grooves from the consumed grains still remain to the right of the small grain as can be seen in the orientation map $(\mathrm{OM})$ in figure $1 \mathrm{~b}$. However, by measuring the misorientation across the boundary relative to the grains and not the sample surface, a misorientation of $\sim 5.0^{\circ}$ exists at every side of the small grain, implying the existence of a low-angle grain boundary. The white pixels in figure $1 \mathrm{~b}$, which were zero solutions in the OM, correspond to the grain boundaries, remnant grooves and pores. It is well known that EBSD sample surfaces should be polished flat and be as clean as possible, [9-10] but this is clearly not possible in such before and after studies where grain boundary grooving is unavoidable. Fluorescence under the electron beam suggested that glass resided both at pores and grain boundaries, giving additional zero solutions in the OM.

A second set of trapped grains was analyzed with EBSD in a similar manner at $70^{\circ}$ tilt. Grains $Y$ and $Z$ in figure $1 \mathrm{c}$ were trapped inside a larger abnormal grain. The abnormal grain has begun to consume grain $Z$, while $Y$ appears to be stable. Misorientation across respective boundaries was characterized using the OM montage in figure $1 \mathrm{~d}$. The zero solutions in the map are again due to fluorescing and sample roughness. The portion of $Z$ consumed by the large grain has the same orientation as the large grain. No subgrains were found. The measured misorientation angles are very close to being a simple rotation about the $c$-axis of the hexagonal unit cell in alumina. 


\section{References}

[1] M.A. O'Keefe, et al., Phil. Mag. B 81(11) (2001) 1861

[2] T. Kamino, et al., Microsc. Microanal. 3 (1997) 393

[3] N. Ravishankar and C.B. Carter, Acta Mater. 49 (2001) 1963

[4] C. Greskovich and J.A. Brewer, J. Am. Ceram. Soc. 84 (2) (2001) 420

[5] M. Hillert, Acta Metall. 13(3) (1965) 238

[6] C.V. Thompson, et al., Acta Metall. 35(4) (1987) 887

[7] J. -K. Park, et al., J. Am. Ceram. Soc. 86(6) (2003) 1014

[8] W.D. Kingery, et al., Introduction to Ceramics, $2^{\text {nd }}$ ed., John Wiley \& Sons, New York, 1976

[9] F.J. Humphreys, Scr. Mater. 51(8) (2004) 771

[10] A.J. Schwartz, et al., Electron Backscatter Diffraction in Materials Science, Kluwer, NY, 2000

[11] This research was supported by the U.S. Department of Energy through grants DE-FG0292ER45465-A004 and DE-FG02-01ER45883 and the 3M Harry Heltzer Endowed Chair. Technical assistance from Dr. Stuart McKernan is gratefully acknowledged.
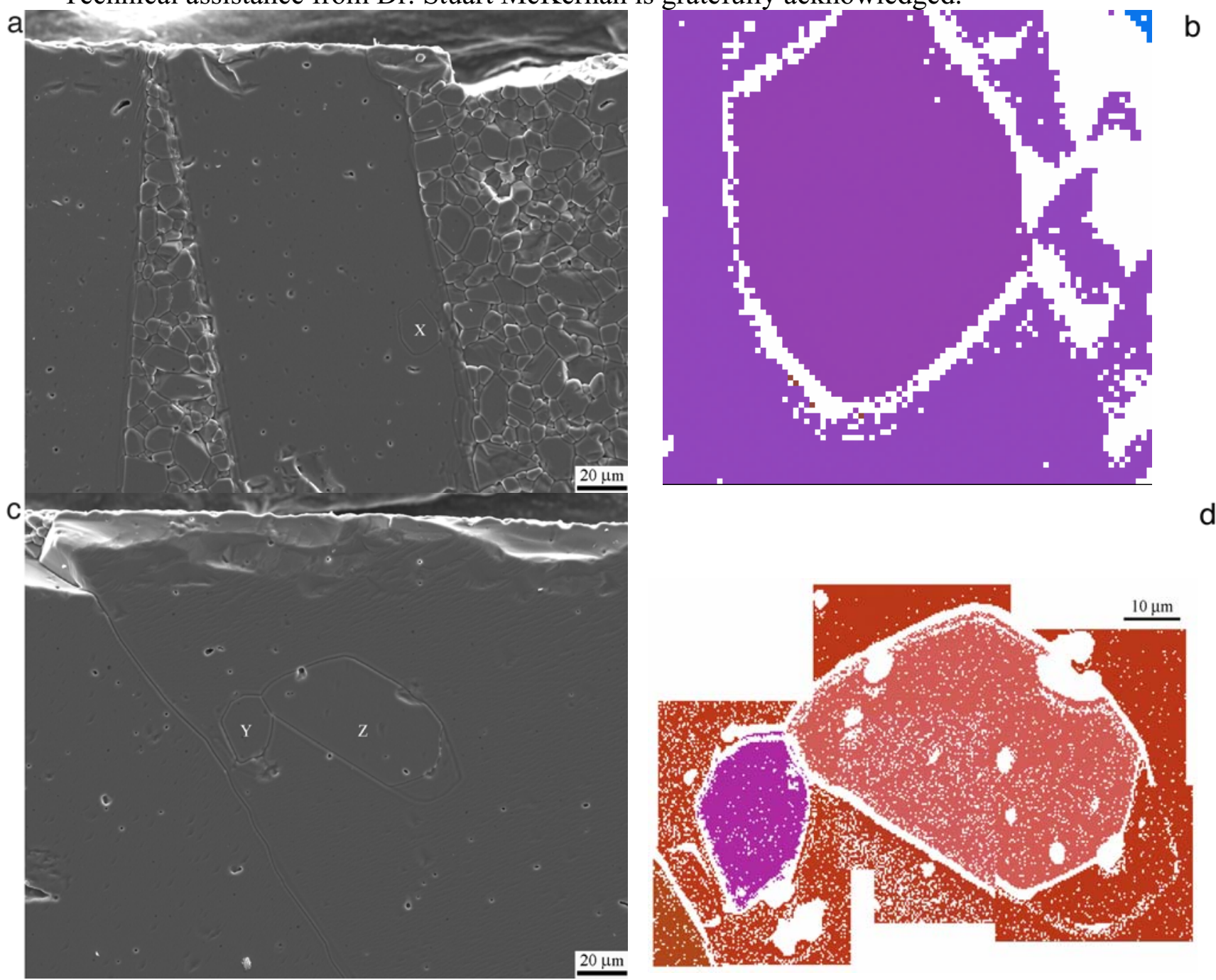

Fig. 1. a) Trapped grain $X$ within an abnormal grain. b) EBSD orientation map of grain $X$ showing a very small misorientation relative to the abnormal grain. Abnormal grains $Y$ and $Z$ trapped inside a larger abnormal grain imaged using c) SE imaging and d) EBSD in the SEM. 